\title{
Metastatic MEN1 Syndrome Treated with Lutetium-177 - A Case Report
}

\author{
Emre Gezer, Berrin Çetinarslan, Zeynep Cantürk, İlhan Tarkun, Mehmet Sözen, Alev Selek \\ Department of Endocrinology and Metabolism, Faculty of Medicine, Kocaeli University, Kocaeli, Turkey
}

$\mathrm{M}$ ultiple endocrine neoplasia type 1 (MEN1) is an autosomal dominant endocrine tumour syndrome characterised by three main manifestations: primary hyperparathyroidism (78-94\%), gastroenteropancreatic neuroendocrine tumours (GEP-NETS) (35-78\%) and pituitary adenomas (20-65\%). For metastatic and inoperable GEP-NETs, there are some interventional and medical therapies. Peptide receptor radionuclide therapy (PRRT) with Yttrium-90 ( ${ }^{\circ} \mathrm{Y}$ ) and Lutetium-177 (17Lu) is one of the important radiotherapies. Herein we describe a case of MEN1 syndrome with inoperable metastatic GEP-NETs who had excellent response to the treatment with six cycles of ${ }^{17}$ Lu-DOTATATE. The patient was admitted to our clinic with widening of hands and feet, polyuria, polydipsia, nausea, vomiting and constipation. His laboratory and screening findings were consistent with primary hyperparathyroidism, acromegaly, secondary hypogonadism and central diabetes insipidus. He underwent 3.5 parathyroidectomy and hypophysis adenomectomy. Under treatment with lanreotide and cabergoline, he developed metastatic duodenal NET. PRRT with 1"LU-DOTATATE was administered in six cycles and an excellent response was displayed without any side effect. In conclusion, the dramatic response of the patient to PRRT with 1"Lu-DOTATATE, described in our case report and recent published articles indicating the beneficial efficacy and limited adverse effects of 1"Lu-DOTATATE, should encourage clinicians to use PRRT for inoperable or metastatic NETs.

\section{Keywords}

MEN1 syndrome, neuroendocrine tumor, metastasis, Lutetium

Disclosures: Emre Gezer, Berrin Çetinarslan, Zeynep Cantürk, Illhan Tarkun, Mehmet Sözen and Alev Selek have nothing to declare in relation to this article. Review Process: Double-blind peer review.

Compliance with Ethics: All procedures were followed in accordance with the responsible committee on human experimentation and with the Helsinki Declaration of 1975 and subsequent revisions, and informed consent was received from the patient involved in this case study.

Authorship: All named authors meet the International Committee of Medical Journal Editors (ICMJE) criteria for authorship of this manuscript, take responsibility for the integrity of the work as a whole, and have given final approval for the version to be published.

Received: 5 March 2019

Accepted: 18 April 2019

Citation: European Endocrinology. 2019;15(2):92-4

Corresponding Author: Emre Gezer, Kocaeli Üniversitesi Tıp Fakültesi Umuttepe Yerleşkesi 41380, izmit/KOCAELi. E: gezer_emre@hotmail.com

Support: No funding was received in the publication of this article.
Multiple endocrine neoplasia type 1 (MEN1) is an autosomal dominant endocrine tumour syndrome characterised by three main manifestations which are primary hyperparathyroidism (78-94\%), gastroenteropancreatic neuroendocrine tumours (GEP-NETS) (35-78\%) and pituitary adenomas (20-65\%). ${ }^{1}$ As described, GEP-NETs are the second most common component of MEN1 syndrome and metastatic GEP-NETs are shown to be related with poor prognosis. ${ }^{2}$

For metastatic and inoperable GEP-NETs, there are some interventional and medical therapies. Peptide receptor radionuclide therapy (PRRT) with Yttrium-90 ( $\left.{ }^{\circ 0} \mathrm{Y}\right)$ and Lutetium-177 (17/LU) is one of the important radiotherapies; however, there have been no randomised controlled study evaluating both efficacy and safety of ${ }^{177}$ Lu-DOTATATE since 2017 . The recently published NETTER-1 study demonstrated the markedly longer progression-free survival (PFS) with ${ }^{177 L u-D O T A T A T E}$ treatment in midgut NETs. ${ }^{3}$

In this case report, we describe a patient with MEN1 syndrome with inoperable metastatic GEP-NETS who had excellent response to the treatment with six cycles of ${ }^{177}$ Lu-DOTATATE.

\section{Case report}

A 35-year-old male patient was admitted to our clinic with widening of hands and feet, polyuria, polydipsia, nausea, vomiting and constipation in 2008. He had a history of hydrocephalus diagnosis when 2 months old. Physical examination showed normal vital signs and findings, except coarsening of voice and large tongue, nose, hands and feet. Laboratory findings revealed low total testosterone $(0.14 \mathrm{ng} / \mathrm{mL})$, luteinising hormone $(0.66 \mathrm{mIU} / \mathrm{mL})$ and follicle stimulating hormone (2.24 mIU/mL); elevated Insulin like growth factor-1 (IGF-1) $(662 \mathrm{ng} / \mathrm{mL}$; according to age- and sex-specific serum IGF-1 levels), growth hormone $(\mathrm{GH})(22.7 \mathrm{ng} / \mathrm{mL})$, and calcium $(11.0 \mathrm{mg} / \mathrm{dl})$; and intact parathyroid hormone concentration $(160 \mathrm{pg} / \mathrm{mL})$ with low phosphate concentration (2.1 mg/dl). Twenty-four-hour urinary calcium excretion was elevated (399 mg/day). Additionally, bone mineral density was also decreased; L1-L4 anteroposterior spine T- and Z-scores were -3.4 SD and -4.2 SD, respectively. Scanning with Technetium-99m-hexakis 2-methoxyisobutyi isonitrile

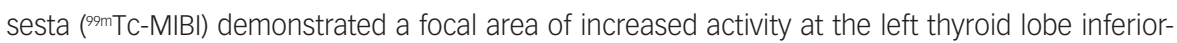
pole region, suggestive of a parathyroid adenoma. These findings, including non-suppressed GH levels by oral glucose tolerance test (OGTT), were compatible with primary hyperparathyroidism, acromegaly and secondary hypogonadism; as a result, he was diagnosed with MEN1 syndrome.

He was also diagnosed with central diabetes insipidus by an overnight water deprivation test. Pituitary magnetic resonance imaging (MRI) revealed a $19 \mathrm{~mm}$ pituitary macroadenoma without compression of optic chiasm and he was operated on by transsphenoidal route. 


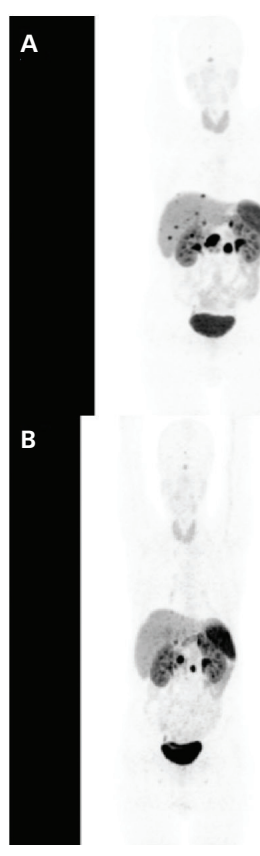

A - Pretreatment Ga68-DOTATATE scan B - Ga68-DOTATATE scan after 2nd cycle
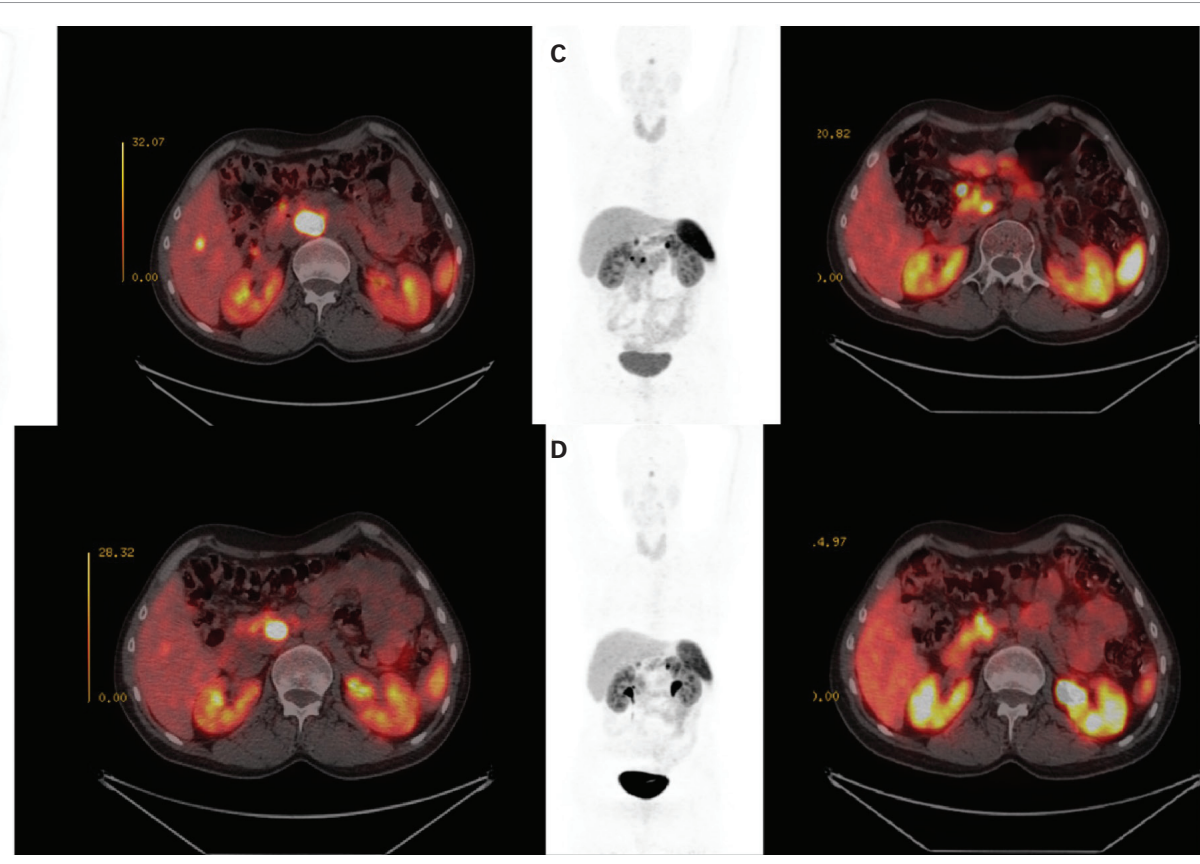

C - Ga68-DOTATATE scan after 4th cycle D - Ga68-DOTATATE scan after 6th cycle

A. Pre-treatment Ga-68-DOTATATE scan; B. Ga-68-DOTATATE scan after second treatment cycle; C. Ga-68-DOTATATE scan after fourth treatment cycle; D. Ga-68-DOTATATE scan after sixth treatment cycle.

Immunhistochemically, the pituitary adenoma was strongly and diffuse stained with $\mathrm{GH}$, focal slightly stained with prolactin. Adrenocorticotrophic hormone staining was not detected. Ki67 index was approximately $8 \%$.

At the patient's follow-up evaluation after surgery, IGF-1 level was still elevated and there was no GH suppression after OGTT. Lanreotide $120 \mathrm{mg} / \mathrm{month}$ and cabergoline treatments were initiated together with desmopressin, and testosterone. Because of the presence of MEN1 syndrome, he also underwent 3.5 parathyroidectomy, as well as adenomectomy, followed by calcium replacement therapy because of postoperative hypocalcaemia. Under treatment with lanreotide and cabergoline for 2 years, the patient's IGF-1 level was still elevated (423 ng/mL) and pituitary MRI was repeated. MRI showed two microadenomas measuring $9 \mathrm{~mm}$ and $7 \mathrm{~mm}$. Surgery was repeated via the transsphenoidal route in 2010. The immunhistopathology report displayed that the pituitary adenoma was stained with $\mathrm{GH}$, and prolactin. Ki67 index was 4\%. Early postoperative MRI showed no residual adenoma. After the operation, disease control was achieved and IGF-1 level was decreased to $89 \mathrm{ng} / \mathrm{mL}$ and stayed within normal range for 2 years. The patient was subsequently lost to follow-up for 2 years.

During routine surveillance of acromegaly from 2010-2012, a liver mass at segment 8 , which enhanced maximally in the early arterial phase and became isodense in the portal venous phase, was recognised on computed tomography (CT) scans. Consequently, abdominal MRI was performed, followed by an octreotide scan, and revealed two focal areas of increased tracer uptake at the wall of duodenum. The liver lesion, which was defined at both $\mathrm{CT}$ and MRI, showed no activity. Gastrin level was also checked, but it was within the range of normal limits. Both duodenal and liver lesions were evaluated as suggestive of a non-functioning NET and its metastases. In addition to that, upper gastrointestinal endoscopy showed multiple lesions at duodenum. Some part of tumour was resected and pathology reported the lesion as grade 1 duodenal NET.
The patient was lost to follow-up for the next 3 years; however, he was referred back to our hospital in 2015. His IGF-1 level was $310 \mathrm{ng} / \mathrm{mL}$, so lanreotide $120 \mathrm{mg} / \mathrm{month}$ was reinitiated. There also appeared liver lesions on abdominal MRI. Thereafter, a Ga-68-DOTATATE scan was performed, which revealed multiple foci with elevated accumulation of Ga-68 (Figure 1). As a result, PRRT with ${ }^{177}$ Lu-DOTATATE was administered in six cycles. Repeated Ga-68-DOTATATE scanning after each two cycles displayed an excellent response to PRRT (Figure 1); additionally, no side effect of this treatment, such as myelosuppression or radiation nephritis, was observed.

\section{Discussion}

PRRT is one of the systemic radiotherapies that allows accumulation of radionuclides in the tumour cells which express high levels of somatostatin receptors. There are two types of radiopeptides used

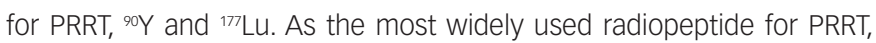
${ }^{177 L U-D O T A T A T E}$ treatment is a valuable therapeutic option for metastasized or inoperable GEP-NETs which harbour the somatostatin receptors on their surface. Inclusion criteria for use of PRRT with ${ }^{177} \mathrm{Lu}$ DOTATATE in NETs is simply presenting an inoperable/metastatic tumour with sufficient tumour uptake on the diagnostic somatostatin receptor scintigraphy. In addition to that, the patient should have $>50 \mathrm{~mL} / \mathrm{min}$ creatinine clearance, $>50$ Karnofsky performance status, sufficient bone marrow reserves and $>3$ months expected survival. ${ }^{4}$

The first-line of treatment for the metastatic GEP-NET in this case may be debatable. There appeared one metastatic lesion on the liver at segment 8 , for which surgery could have also been considered. Chakedis et al. examined 581 patients with metastatic NETs in various organs including the liver (61.3\%), who underwent resection. They reported that removing all metastatic disease had better prognosis than debulking or palliative resection $(112.5,89.2$ and 50.0 months, respectively, $p<0.001) .{ }^{5}$ Despite these promising results, the multidisciplinary council at our clinic 
decided to use ${ }^{177}$ Lu-DOTATATE for the management of the metastatic disease. The primary reason for this consensus was the elevated accumulation of Ga-68 at described lesions and the young age of our patient. A systemic therapy, not as risky as surgery, was considered first and if it failed, the surgery would be an alternative option.

As described earlier, the NETTER-1 study is the first randomised phase III study to compare ${ }^{177} \mathrm{Lu}$-DOTATATE to high-dose octreotide long-acting repeatable (LAR) therapy in patients with inoperable and progressive midgut NETs. ${ }^{6}$ In this trial, 229 patients with inoperable, metastatic, well-differentiated somatostatin-receptor-positive midgut NETs who had progressive disease on standard dose (30 mg every 3-4 weeks) of octreotide LAR were included. They were randomised to an additional four cycles of ${ }^{177} \mathrm{Lu}$-DOTATATE (200 mCi each) every 8 weeks or octreotide LAR $60 \mathrm{mg}$ alone every 28 days (control group). The estimated PFS rate at month 20 was $65.2 \%$ in the ${ }^{177}$ Lu-DOTATATE group and $10.8 \%$ in the control group. These results clearly indicated that the treatment with ${ }^{177}$ Lu-DOTATATE had a significantly higher response rate than high-dose octreotide LAR among patients with advanced midgut
NETS. Another promising outcome was the limited clinically significant myelosuppression rate with ${ }^{177}$ Lu-DOTATATE treatment which was less than $10 \%$ of patients.

Furthermore, one of the largest studies which involved over 1,200 patients with advanced grade 1-2 GEP or lung NETs (with a Ki67 index of $20 \%$ or below) treated with ${ }^{17}$ Lu-DOTATATE demonstrated the excellent efficacy and limited transient side effects of this therapeutic option. There was no therapy-related long-term renal or hepatic failure. ${ }^{6}$ In another study of 265 patients with NETs reported in 2011, it was stated that the quality of life improved significantly after ${ }^{177}$ Lu-DOTATATE therapy.?

In conclusion, the dramatic response to PRRT with ${ }^{17} \mathrm{Lu}$-DOTATATE described in this case report, and recent published articles indicating the beneficial efficacy and limited adverse effects of 177 Lu-DOTATATE, should encourage clinicians to use PRRT for inoperable or metastatic NETs. Complete blood count, renal function tests, performance status of the patient and somatostatin receptor scintigraphy images should be assessed cautiously before initiating this treatment.
1. Pieterman CRC, Vriens MR, Dreijerink KMA, et al. Care for patients with multiple endocrine neoplasia type 1: The current evidence base. Fam Cancer. 2011;10:157-71.

2. Ito $T$, Igarashi $H$, Uehara $H$, et al. Causes of death and prognostic factors in multiple endocrine neoplasia type 1: A prospective study: Comparison of 106 men1/zollinger-ellison syndrome patients with 1613 literature men1 patients with or without pancreatic endocrine tumors. Medicine (Baltimore) 2013;92:135-81.

3. Strosberg J, El-Haddad G, Wolin E, et al. Phase 3 trial of
${ }^{177} \mathrm{~L}$ U-Dotatate for midgut neuroendocrine tumors. N Eng/ J Med. 2017:376:125-35.

4. Hicks RJ, Kwekkeboom DJ, Krenning E, et al. ENETS consensus guidelines for the standards of care in neuroendocrine neoplasms: peptide receptor radionuclide therapy with radiolabelled somatostatin analogues. Neuroendocrinology. 2017; 105:295-309.

5. Chakedis J, Beal EW, Lopez-Aguiar AG, et al. Surgery provides long-term survival in patients with metastatic neuroendocrine tumors undergoing resection for non-hormonal symptoms $/$ Gastrointest Surg. 2019;23:122-34.

6. Brabander T, Van Der Zwan WA, Teunissen JJM, et al. Long-term efficacy, survival, and safety of $\left[{ }^{177}\right.$ Lu-DOTA ${ }^{0}$, Tyr $\left.r^{3}\right]$ octreotate in patients with gastroenteropancreatic and bronchial neuroendocrine tumors. Clin Cancer Res. 2017;23:4617-24.

7. Khan S, Krenning EP, van Essen M, Kam BL, et al. Quality of life in 265 patients with gastroenteropancreatic or bronchial neuroendocrine tumors treated with [177Lu-DOTA0,Tyr3] octreotate. J Nucl Med. 2011;52:1361-8. 